\title{
Finite-Time Boundedness of Markov Jump System with Piecewise-Constant Transition Probabilities via Dynamic Output Feedback Control
}

\author{
Bin Yan, ${ }^{1}$ Xiaojia Zhou, ${ }^{1}$ Jun Cheng, ${ }^{2}$ and Fangnian Lang ${ }^{3}$ \\ ${ }^{1}$ School of Automation Engineering, University of Electronic Sciences and Technology of China, Chengdu 611731, China \\ ${ }^{2}$ School of Information, Hubei University for Nationalities, Enshi 445000, China \\ ${ }^{3}$ School of Computer, Chengdu University, Chengdu 610106, China \\ Correspondence should be addressed to Bin Yan; uestyan@uestc.edu.cn
}

Received 14 March 2015; Accepted 28 April 2015

Academic Editor: Leonid Shaikhet

Copyright (C) 2015 Bin Yan et al. This is an open access article distributed under the Creative Commons Attribution License, which permits unrestricted use, distribution, and reproduction in any medium, provided the original work is properly cited.

\begin{abstract}
This paper first investigates the problem of finite-time boundedness of Markovian jump system with piecewise-constant transition probabilities via dynamic output feedback control, which leads to both stochastic jumps and deterministic switches. Based on stochastic Lyapunov functional, the concept of finite-time boundedness, average dwell time, and the coupling relationship among time delays, several sufficient conditions are established for finite-time boundedness and $H_{\infty}$ filtering finite-time boundedness. The system trajectory stays within a prescribed bound. Finally, an example is given to illustrate the efficiency of the proposed method.
\end{abstract}

\section{Introduction}

Markovian jump systems were introduced as a class of stochastic switched systems, which can be governed by a Markov chain in a finite mode set of linear dynamics. In recent years, because it is appropriate to model many physical systems with economics, random failures, and networked control systems, more and more people draw their attention to Markovian jump systems [1-4]. As a special class of stochastic systems in the finite operation modes, Markovian jump systems can switch from one to another at different time. Up to now, many important results in the literature are based on the assumption that the complete knowledge of transition probabilities is available in the jump process. However, at mode observation instants, the Markovian jump modes of the systems cannot be accurately obtained, and to get the ideal information on all transition rates is hard or generally expensive in reality, and the obtained results are not accurate. Therefore, it is very important to consider systems based on the assumption that transition probabilities are completely unknown. Recently, the Markovian jump systems subject to partially known transition probabilities have been reported [5-10]. However, the Markov processes are timeinvariant in most of aforementioned obtained results.

Nowadays, piecewise-homogeneous (namely, timevarying transition probabilities) Markovian jump systems are developed for practical applications, affecting not only the time-varying transition probabilities but also the state dynamics. The evolution between two operating modes with time-varying transition probabilities was proposed in economy systems [11, 12]. Because of the important issue of the possibility in measuring the variations, up till now, a few people in view of stochastic Markovian jump systems with time-varying transition probabilities except in [13-19]. In [14], there is a bounded real lemma for Markovian jump linear systems with time-varying transition probabilities in discrete-time domain. The Markov switching is employed for sustainability of US external debt in [15]. The linear matrix inequalities are used for control theory in Markov switching [16]. In [19], newly Lyapunov functional is proposed with piecewise-constant transition probabilities. It should be noted that average dwell time switching is very important in dynamic systems [20-23]. In [20], the average dwell time switching and uncertainties are considered. Correspondingly, 
a dependent average dwell time approach is proposed in [21]. The piecewise-homogeneous is taken into account which makes the considering dynamic of the Markovian jump systems more controllable and optimizes the performance of systems.

Furthermore, in a finite horizon, the practical application problems tend to care about the described systems' transient characteristics state, especially the transient performances of control systems. It is necessary to consider the state in a fixed region; therefore, the concept of finite-time stability was introduced $[22,23]$. Some research results in finite-time case for Markovian jump systems can be found in [24-30]. For example, the finite-time stabilization with output feedback control is introduced in [24]. Finite-time boundedness is considered with state-dependent switching strategy in [26]. In [27], finite-time $H_{\infty}$ control is proposed for nonlinear jump systems. In [29], the partially unknown transition rates are introduced for finite-time filtering of stochastic systems. It is noted that, in the engineering area, there are still some problems related to stochastic systems to be solved. In order to make the finite-time behaviour of stochastic Markovian jump systems more reasonable and satisfy the requirements, the finite-time boundedness of Markov jump systems with piecewise-constant transition probabilities via dynamic output feedback control has not been studied. The problem is interesting but also challenging, which motivates us to conduct this study.

The main contribution of this paper is that we present a novel approach for finite-time boundedness of Markovian jump system with piecewise-constant transition probabilities via dynamic output feedback control. We establish a more general model to extend the existing results into more feedback control systems. The deterministic switches and stochastic jumps are taken into account at the same times. The finite-time stability is an independent concept, which is different from Lyapunov stability and can be determined by switching. By selecting the appropriate Lyapunov-Krasovskii functional, under average dwell time constraint on switching signals, the sufficient conditions among average dwell times, transition probabilities, and time-varying delay are derived to guarantee finite-time boundedness of the Markovian jump systems.

\section{Preliminaries}

In this paper, fixing the probability space $(\Omega, \mathscr{F}, \mathscr{P})$, we consider the following Markovian jump system described by

$$
\begin{aligned}
& \dot{x}(t)=A_{r_{t}} x(t)+A_{\tau r_{t}} x(t-\tau)+B_{r_{t}} u(t)+D_{r_{t}} \omega(t), \\
& y(t)=C_{r_{t}} x(t), \\
& x(t)=\phi(t),
\end{aligned}
$$

$$
t \in[-\tau, 0]
$$

where $x(t) \in \mathscr{R}^{n}$ is the state vector of the system, $y(t) \in \mathscr{R}^{l}$ is the measured output, and $\omega(t) \in L_{2}^{q}[0, \infty]$ is the exogenous noise signal. $A_{r_{t}}, A_{\tau r_{t}}, B_{r_{t}}, D_{r_{t}}$, and $C_{r_{t}}$ are constant real matrices with appropriate dimension. $\tau$ represent the constant delay and $\phi(t)$ is the differentiable vector-valued initial function on $[-\tau, 0]$. Let the random form process $r_{t}, t \geq 0$ be the Markov stochastic process taking values on a finite set $\mathcal{N}=\{1,2, \ldots, N\}$, governing the switching from mode $i$ at time $t$ to mode $j$ at time $t+\Delta t$ with the following transition probabilities:

$$
\begin{aligned}
P_{i j} & =\operatorname{Pr}\left(r_{t+\Delta t}=j \mid r_{t}=i\right) \\
& = \begin{cases}\mu_{i j}^{\left(\sigma_{t}\right)} \Delta t+o(\Delta t), & i \neq j, \\
1+\mu_{i i}^{\left(\sigma_{t}\right)} \Delta t+o(\Delta t), & i=j,\end{cases}
\end{aligned}
$$

with transition rates $\mu_{i j}^{\left(\sigma_{t}\right)} \geq 0, \forall i, j \in \mathcal{N}, \sum_{j=1}^{N} \mu_{i j}^{\left(\sigma_{t}\right)}=0$, $\Delta t>0$, and $\lim _{\Delta t \rightarrow 0}(o(\Delta t) / \Delta t) \rightarrow 0$. Here, $\mu_{i j}^{\left(\sigma_{t}\right)}$ is now a function of $\sigma_{t}$. By $\sigma_{t}$, we mean that the transition rates are time-varying. Moreover, $\sigma_{t}$ is assumed to be piecewiseconstant function of time $t$, and transition rates $\Pi^{\sigma_{t}}$ can be defined by

$$
\Pi^{\sigma_{t}}=\left[\begin{array}{cccc}
\mu_{11}^{\left(\sigma_{t}\right)} & \mu_{12}^{\left(\sigma_{t}\right)} & \cdots & \mu_{1 N}^{\left(\sigma_{t}\right)} \\
\mu_{21}^{\left(\sigma_{t}\right)} & \mu_{22}^{\left(\sigma_{t}\right)} & \cdots & \mu_{2 N}^{\left(\sigma_{t}\right)} \\
\vdots & \vdots & \vdots & \vdots \\
\mu_{N 1}^{\left(\sigma_{t}\right)} & \mu_{N 2}^{\left(\sigma_{t}\right)} & \cdots & \mu_{N N}^{\left(\sigma_{t}\right)}
\end{array}\right] .
$$

Furthermore, to determine the time-varying property, $\sigma_{t}$ represents a high-level average dwell time switching signal. $\sigma_{t}$ is a given initial condition sequence. For simplicity, let $m$ represent $\sigma_{t}$ as a piecewise-constant function of time, which takes values in the finite set $\mathscr{M} \equiv\{1,2, \ldots, M\}$. At an arbitrary time $t, \sigma$ may be dependent on $t$ or $x(t)$, or both, or other logic rules. For a switching sequence $t_{0}<t_{1}<t_{2}<\cdots$, $\sigma$ is continuous from right everywhere and maybe either autonomous or controlled. When $k \in\left[t_{l}, t_{l+1}\right)$, we say that the $\sigma_{t_{l}}$ th transition probabilities matrix is active and therefore the trajectory $x_{t}$ of system (1) is trajectory of system (1) with the $\sigma_{t_{l}}$ th transition probabilities matrix.

In this paper, our goal is to design the following dynamic output feedback controller, which can guarantee the system is finite-time boundness:

$$
\begin{aligned}
\dot{x}_{f}(t) & =A_{f r_{t}, \sigma_{t}} x_{f}(t)+B_{f r_{t}, \sigma_{t}} y(t), \\
u(t) & =C_{f r_{t}, \sigma_{t}} x_{f}(t)+D_{f r_{t}, \sigma_{t}} y(t), \\
x_{f}(t) & =0,
\end{aligned}
$$

$$
t \leq 0
$$

where $A_{f r_{t}, \sigma_{t}}, B_{f r_{t}, \sigma_{t}}, C_{f r_{t}, \sigma_{t}}$, and $D_{f r_{t}, \sigma_{t}}$ are matrices to be determined. 
Substituting (4) into (1) and $\forall r_{t}=i, i \in \mathcal{N}, \sigma_{t}=m$, and $m \in \mathscr{M}$, we have

$$
\begin{aligned}
\dot{\eta}(t) & =\bar{A}_{i, m} \eta(t)+\bar{A}_{\tau i} \eta(t-\tau)+\bar{B}_{i} \omega(t), \\
\eta(t) & =\psi(t),
\end{aligned}
$$

$$
t \in[-\tau, 0]
$$

where

$$
\begin{aligned}
\bar{A}_{i, m} & =\left[\begin{array}{cc}
A_{i}+B_{i} D_{f i, m} C_{i} & B_{i} C_{f i, m} \\
B_{f i, m} C_{i} & A_{f i, m}
\end{array}\right], \\
\bar{A}_{\tau i} & =\left[\begin{array}{cc}
A_{\tau i} & 0 \\
0 & 0
\end{array}\right], \\
\bar{B}_{i} & =\left[\begin{array}{c}
D_{i} \\
0
\end{array}\right], \\
\eta(t) & =\left[\begin{array}{c}
x(t) \\
x_{f}(t)
\end{array}\right] .
\end{aligned}
$$

Throughout the paper, suppose that the matrices $C_{r_{t}}$ have full row rank, in other words, $\operatorname{rank}\left(C_{r_{t}}\right)=q$. Then we have the singular decomposition of $C_{i}$ as

$$
C_{i}=U_{i}\left[S_{i}, 0\right] V_{i}^{\top},
$$

where $S_{i} \in R^{q \times q}$ is a diagonal positive matrix and $U_{i} \in R^{q \times q}$ and $V_{i} \in R^{n \times n}$ are unitary matrices.

Remark 1. In this paper, matrices $C_{i}$ are singular decomposition as unitary matrices, which reduce the conservatism.

First of all, we will give definitions and lemmas about system (5), which plays an important role in the derivation of our result.

Definition 2 (see [29]). System (5) is said to be finite-time bounded with respect to $\left(c_{1}, c_{2}, T, R, d, \sigma\right)$, where $d \geq 0, R$ is positive define matrix, and $\sigma_{t}$ is a switching signal. We have

$$
\begin{aligned}
& \sup _{-\tau \leq t_{0} \leq 0}\left\{x^{\top}\left(t_{0}\right) R x\left(t_{0}\right)\right\} \\
& \quad \leq c_{1} \Longrightarrow \sup _{-\tau \leq t \leq 0}\left\{x^{\top}(t) R x(t)\right\}<c_{2}, \quad \forall t \in[0, T],
\end{aligned}
$$

where $c_{2}>c_{1} \geq 0, \forall \omega(t): \int_{0}^{T} \omega^{\top}(s) \omega(s) d s \leq d$.

Definition 3 (see [21]). For any $T_{2}>T_{1} \geq 0$, let $N_{\sigma}\left(T_{1}, T_{2}\right)$ denote the switching number of $\sigma(t)$ during $\left(T_{1}, T_{2}\right)$. If $N_{\sigma}\left(T_{1}, T_{2}\right) \leq N_{0}+\left(T_{2}-T_{1}\right) / T_{a}$ holds for $N_{0} \geq 0$ and $T_{a}>0$, then $N_{0}$ and $T_{a}$ are called chattering bound and average dwell time, respectively. Here we assume $N_{0}=0$ for simplicity as commonly used in the literature.
Definition 4 (see [31]). Consider $V\left(\eta_{t}, r_{t}, \sigma_{t}\right)$ as the stochastic Lyapunov function of the resulting system (4); its weak infinitesimal operator is defined as

$$
\begin{aligned}
£ V & \left(\eta_{t}, r_{t}, \sigma_{t}\right) \\
& =\lim _{\Delta t \rightarrow 0^{+}} \frac{1}{\Delta t}\left[\mathbb{E}\left\{V\left(\eta_{t+\Delta t}, r_{t+\Delta t}, \sigma_{t}\right) \mid r_{t}=i, \sigma_{t}=m\right\}\right. \\
& \left.-V\left(\eta_{t}, i, m\right)\right]=\frac{\partial}{\partial t} V\left(\eta_{t}, i, m\right)+\frac{\partial}{\partial x} V\left(\eta_{t}, i, m\right) \dot{\eta}_{t} \\
& +\sum_{j=1}^{N} \mu_{i j}^{(m)} V\left(\eta_{t}, j, m\right) .
\end{aligned}
$$

Definition 5 (see [32]). The jump rates of the visited modes from a given mode $i$ are assumed to satisfy

$$
0<\min \mu_{i} \leq \mu_{i j} \leq \max \mu_{i}, \quad \forall i, j \in \mathbb{R}, i \neq j,
$$

where $\min \mu_{i}$ and $\max \mu_{i}$ are known parameters for a given mode $i$ and represent the lower and upper bounds when all the jump rates are known; that is, $0<\min \mu_{i}=\min \left\{\mu_{i j} \neq\right.$ $0, i \neq j, j \in \mathbb{R}\}$ and $\min \mu_{i} \leq \max \mu_{i}$. Meanwhile, the number of the visited modes from a given mode $i$ is denoted by $N_{i}$ including the mode itself.

Lemma 6 (Schur complement [14]). Given constant matrices $X, Y$, and $Z$, where $X=X^{\top}$ and $0<Y=Y^{\top}$, then $X+Z^{\top} Y^{-1} Z<0$ if and only if

$$
\begin{array}{r}
{\left[\begin{array}{cc}
X & Z^{\top} \\
* & -Y
\end{array}\right]<0} \\
\text { or }\left[\begin{array}{cc}
-Y & Z \\
* & X
\end{array}\right]<0 .
\end{array}
$$

\section{Finite-Time Boundedness Analysis}

Theorem 7. System (5) is finite-time stochastic boundedness (FTSB) with respect to $\left(c_{1}, c_{2}, R, d, T\right)$ if there exist matrices $P_{i, m}, H$, and $Q_{m}$ and constants $\alpha \geq 0, \mu>1$, and $\lambda_{s}>0$ $(s=1,2, \ldots, 4)$, such that we have the following linear matrix inequalities:

$\left[\begin{array}{ccc}\bar{A}_{i, m}^{\top} P_{i, m}^{-1}+P_{i, m}^{-1} \bar{A}_{i, m}+Q_{m}^{-1}+\bar{P}_{i, m}-\alpha P_{i, m}^{-1} & P_{i, m}^{-1} \bar{A}_{\tau i} & P_{i, m}^{-1} \bar{B}_{i} \\ * & -e^{\alpha \tau} Q_{m}^{-1} & 0 \\ * & * & -\alpha H\end{array}\right]$

$<0$,

$\left[\begin{array}{ccc}-\lambda_{1} c_{2} e^{-\alpha T}+d \lambda_{4}\left(1-e^{-\alpha T}\right) & \lambda_{2} c_{1} & \lambda_{3} c_{1} \\ * & -\lambda_{2} c_{1} & 0 \\ * & * & -\frac{1}{\tau} \lambda_{3} c_{1} e^{-\alpha \tau}\end{array}\right]<0$, 


$$
\begin{aligned}
& {\left[\begin{array}{cc}
-P_{i, m}^{-1} & \lambda_{1} P_{i, m}^{-1} R \\
* & -\lambda_{1} R
\end{array}\right]<0,} \\
& {\left[\begin{array}{cc}
-\lambda_{2} R & I \\
* & -P_{i, m}^{-1}
\end{array}\right]<0,} \\
& P_{i, m}<\mu P_{i, n}, \\
& Q_{m} \leq Q_{n}, \\
& \lambda_{1} c_{2} e^{-\alpha T}>\left(\lambda_{2}+\tau e^{\alpha \tau} \lambda_{3}\right) c_{1}+d \lambda_{4}\left(1-e^{-\alpha T}\right),
\end{aligned}
$$

with the average dwell time of the switching signal $\sigma$ satisfying

$$
\begin{aligned}
& \tau_{a} \\
& >\tau_{a}^{*} \\
& =\frac{T \ln \mu}{\ln \left(\lambda_{1} c_{2}\right)-\ln \left[\left(\lambda_{2}+\tau e^{\alpha \tau} \lambda_{3}\right) c_{1}+d \lambda_{4}\left(1-e^{-\alpha T}\right)\right]-\alpha T},
\end{aligned}
$$

where

$$
\begin{aligned}
\bar{P}_{i, m}= & -\left(N_{i}-1\right)\left(\min \mu_{i}^{m}\right) P_{i, m} \\
& +\left(\max \mu_{i}^{m}\right) \sum_{j=1, j \neq i}^{N} P_{j, m}, \\
\widetilde{P}_{i, m}= & R^{-1 / 2} P_{i, m} R^{-1 / 2}, \\
\widetilde{Q}_{m}= & R^{-1 / 2} Q_{m} R^{-1 / 2}, \\
\lambda_{3}= & \lambda_{\max }\left(Q_{m}\right), \\
\lambda_{4}= & \lambda_{\max }(H) .
\end{aligned}
$$

Proof. We consider the following Lyapunov-Krasovskii functional:

$$
V\left(\eta_{t}, r_{t}, \sigma_{t}\right)=\eta_{t}^{\top} P_{r_{t}, \sigma_{t}}^{-1} \eta_{t}+\int_{t-\tau}^{t} \eta_{s}^{\top} e^{\alpha(t-s)} Q_{\sigma_{s}}^{-1} \eta_{s} d s
$$

Taking the time derivative of $V\left(\eta_{t}, r_{t}, \sigma(t)\right)$ along the trajectory of the system (5), one has

$$
\begin{aligned}
£ V & \left(\eta_{t}, r_{t}, \sigma_{t}\right) \\
= & \eta_{t}^{\top}\left(\bar{A}_{i, m}^{\top} P_{i, m}^{-1}+P_{i, m}^{-1} \bar{A}_{i, m}+Q_{m}^{-1}+\sum_{j=1}^{N} \mu_{i j}^{(m)} P_{j, m}\right) \eta_{t} \\
& +2 \eta_{t}^{\top} P_{i, m}^{-1} \bar{A}_{\tau i, m} \eta_{t-\tau}+2 \eta_{t}^{\top} P_{i, m}^{-1} \bar{B}_{i} \omega_{t} \\
& -e^{\alpha \tau} \eta_{t-\tau}^{\top} Q_{m}^{-1} \eta_{t-\tau}+\alpha \int_{t-\tau}^{t} \eta_{s}^{\top} e^{\alpha(t-s)} Q_{\sigma_{s}}^{-1} \eta_{s} d s
\end{aligned}
$$

Moreover, we have

$$
\sum_{j=1}^{N} \mu_{i j}^{(m)} P_{j, m}^{-1}=\mu_{i i}^{m} P_{i, m}^{-1}+\sum_{j=1, j \neq i}^{N} \mu_{i j}^{(m)} P_{j, m}^{-1}
$$

$$
\begin{aligned}
= & -\sum_{j=1, j \neq i}^{N} \mu_{i j}^{(m)} P_{i, m}^{-1}+\sum_{j=1, j \neq i}^{N} \mu_{i j}^{(m)} P_{j, m}^{-1} \\
\leq & -\left(N_{i}-1\right)\left(\min \mu_{i}^{m}\right) P_{i, m}^{-1} \\
& +\left(\max \mu_{i}^{m}\right) \sum_{j=1, j \neq i}^{N} P_{j, m}^{-1} .
\end{aligned}
$$

Assuming that condition (12) is satisfied, we obtain

$$
£ V\left(\eta_{t}, r_{t}, \sigma_{t}\right)-\alpha V\left(\eta_{t}, r_{t}, \sigma_{t}\right)<\alpha \omega_{t}^{\top} H \omega_{t}
$$

Notice that

$$
\frac{d}{d t}\left(e^{-\alpha t} V\left(\eta_{t}, r_{t}, \sigma_{t}\right)\right)<\alpha e^{-\alpha t} \omega_{t}^{\top} H \omega_{t}
$$

Integrate (23) from $t_{k}$ to $t$, from which we can get that

$$
\begin{array}{r}
V\left(\eta_{t}, r_{t}, \sigma_{t}\right)<e^{\alpha\left(t-t_{k}\right)} V\left(\eta_{t_{k}}, r_{t_{k}}, \sigma_{t_{k}}\right) \\
+\alpha \int_{t_{k}}^{t} e^{\alpha(t-s)} \omega_{s}^{\top} H \omega_{s} d s
\end{array}
$$

Noting that $\forall t \in\left[t_{k}, t_{k+1}\right]$, where $t_{k}$ is the $k$ th switching instant and $x_{t_{k}}=x_{t_{k}^{-}}$, from condition (15) it yields

$$
V\left(\eta_{t_{k}}, r_{t_{k}}, \sigma_{t_{k}}\right) \leq \mu V\left(\eta_{t_{k}^{-}}, r_{t_{k}^{-}}, \sigma_{t_{k}^{-}}\right)
$$

From condition (24) and (25), we can easily have

$$
\begin{aligned}
V\left(\eta_{t_{k}}, r_{t_{k}}, \sigma_{t_{k}}\right)< & \mu e^{\alpha\left(t-t_{k}\right)} V\left(\eta_{t_{k}^{-}}, r_{t_{k}^{-}}, \sigma_{t_{k}^{-}}\right) \\
& +\alpha \mu \int_{t_{k}}^{t} e^{\alpha(t-s)} \omega_{s}^{\top} H \omega_{s} d s .
\end{aligned}
$$

Thus, from (24)-(26), it yields

$$
\begin{gathered}
V\left(\eta_{t}, r_{t}, \sigma_{t}\right) \leq e^{\alpha\left(t-t_{k}\right)} V\left(\eta_{t_{k}}, r_{t_{k}}, \sigma_{t_{k}}\right) \\
+\alpha \int_{t_{k}}^{t} e^{\alpha(t-s)} \omega_{s}^{\top} H \omega_{s} d s
\end{gathered}
$$




$$
\begin{array}{lr}
\leq \mu e^{\alpha\left(t-t_{k-1}\right)} V\left(\eta_{t_{k-1}^{-}}, r_{t_{k-1}^{-}}, \sigma_{t_{k-1}^{-}}\right) & \text {Thus } \\
+\alpha \mu \int_{t_{k-1}}^{t_{k}} e^{\alpha(t-s)} \omega_{s}^{\top} H \omega_{s} d s+\alpha \int_{t_{k}}^{t} e^{\alpha(t-s)} \omega_{s}^{\top} H \omega_{s} d s & V\left(\eta_{t}, r_{t}, \sigma_{t}\right) \leq \mu^{T / \tau_{a}} e^{\alpha T}\left\{\left(\lambda_{2}+\tau e^{\alpha \tau} \lambda_{3}\right) c_{1}\right. \\
\leq \mu^{2} e^{\alpha\left(t_{k}-t_{k-2}\right)} V\left(\eta_{t_{k-2}^{-}}, r_{t_{k-2}^{-}}, \sigma_{t_{k-2}^{-}}\right) & \left.+d \lambda_{4}\left(1-e^{-\alpha T}\right)\right\} \\
+\alpha \mu^{2} \int_{t_{k-2}}^{t_{k-1}} e^{\alpha(t-s)} \omega_{s}^{\top} H \omega_{s} d s & =e^{\left(\alpha+\left((\ln \mu) / \tau_{a}\right)\right) T}\left\{\left(\lambda_{2}+\tau e^{\alpha \tau} \lambda_{3}\right) c_{1}\right. \\
\end{array}
$$$$
+\alpha \mu \int_{t_{k-1}}^{t_{k}} e^{\alpha(t-s)} \omega_{s}^{\top} H \omega_{s} d s+\alpha \int_{t_{k}}^{t} e^{\alpha(t-s)} \omega_{s}^{\top} H \omega_{s} d s
$$$$
\leq \cdots \leq \mu^{N_{\sigma}(0, t)} e^{\alpha t} V\left(\eta_{t_{0}}, r_{t_{0}}, \sigma_{t_{0}}\right)
$$$$
+\alpha \mu^{N_{\sigma}(0, t)} \int_{0}^{t_{1}} e^{\alpha(t-s)} \omega_{s}^{\top} H \omega_{s} d s
$$$$
+\alpha \mu^{N_{\sigma}\left(t_{1}, t\right)} \int_{t_{1}}^{t_{2}} e^{\alpha(t-s)} \omega_{s}^{\top} H \omega_{s} d s+\cdots
$$$$
+\alpha \int_{t_{k}}^{t} e^{\alpha(t-s)} \omega_{s}^{\top} H \omega_{s} d s=\mu^{N_{\sigma}(0, t)} e^{\alpha t} V\left(\eta_{t_{0}}, r_{t_{0}}, \sigma_{t_{0}}\right)
$$$$
+\alpha \int_{0}^{t} e^{\alpha(t-s)} \mu^{N_{\sigma}(s, t)} \omega_{s}^{\top} H \omega_{s} d s
$$$$
\leq \mu^{N_{\sigma}(0, t)} e^{\alpha t} V\left(\eta_{t_{0}}, r_{t_{0}}, \sigma_{t_{0}}\right)+\alpha \mu^{N_{\sigma}(0, t)} d \lambda_{\max }(H)
$$$$
\cdot e^{\alpha t} \int_{0}^{t} e^{-\alpha s} d s \leq \mu^{N_{\sigma}(0, T)} e^{\alpha T}\left\{V\left(\eta_{t_{0}}, r_{t_{0}}, \sigma_{t_{0}}\right)\right.
$$$$
\left.+d \lambda_{\max }(H) \alpha \int_{0}^{T} e^{-\alpha s} d s\right\}
$$$$
\leq \mu^{T / \tau_{a}} e^{\alpha T}\left\{V\left(\eta_{t_{0}}, r_{t_{0}}, \sigma_{t_{0}}\right)\right.
$$$$
\left.+d \lambda_{\max }(H)\left(1-e^{-\alpha T}\right)\right\} \text {. }
$$

On the other hand

$$
V\left(\eta_{t}, r_{t}, \sigma_{t}\right) \geq \lambda_{\max }\left(\widetilde{P}_{r_{t}, \sigma_{t}}\right) \eta_{t}^{\top} R \eta_{t}=\lambda_{1} \eta_{t}^{\top} R \eta_{t}
$$

Substituting (29) and (30) into (19), one obtains

$x^{\top}(t) R x(t)$

$$
\leq \frac{\left(\lambda_{2}+\tau e^{\alpha \tau} \lambda_{3}\right) c_{1}+d \lambda_{4}\left(1-e^{-\alpha T}\right)}{\lambda_{1}} e^{\left(\alpha+\left((\ln \mu) / \tau_{a}\right)\right) T} .
$$

When $\mu=1$, which is the trivial case, from (17), $\eta_{t}^{\top} R x_{t}<$ $c_{2} e^{-\alpha T} e^{\alpha T}=c_{2}$. When $\mu>1$, from (17), $\ln \left(\lambda_{1} c_{2}\right)-\ln \left[\left(\lambda_{2}+\right.\right.$ $\left.\left.\tau e^{\alpha \tau} \lambda_{3}\right) c_{1}+d \lambda_{4}\left(1-e^{-\alpha T}\right)\right]-\alpha T>0$ we have

$$
\begin{aligned}
& \frac{T_{f}}{\tau_{a}} \\
& <\frac{\ln \left(\lambda_{1} c_{2}\right)-\ln \left[\left(\lambda_{2}+\tau e^{\alpha \tau} \lambda_{3}\right) c_{1}+d \lambda_{4}\left(1-e^{-\alpha T}\right)\right]-\alpha T}{\ln \mu} \\
& =\frac{\ln \left(\lambda_{1} c_{2} e^{-\alpha T} /\left(\left(\lambda_{2}+\tau e^{\alpha \tau} \lambda_{3}\right) c_{1}+d \lambda_{4}\left(1-e^{-\alpha T}\right)\right)\right)}{\ln \mu} .
\end{aligned}
$$

Substituting (32) into (31) yields

$$
\eta_{t}^{\top} R \eta_{t}<c_{2}
$$

$$
\begin{aligned}
V( & \left.\eta_{t_{0}}, r_{t_{0}}, \sigma_{t_{0}}\right) \\
= & \eta_{t_{0}}^{\top} P_{r_{t_{0}}, \sigma\left(t_{0}\right)} \eta_{t_{0}}+\int_{-\tau}^{0} \eta_{s}^{\top} e^{-\alpha s} Q_{\sigma_{s}} \eta_{s} d s \\
\leq & \lambda_{\max }\left(\widetilde{P}_{i, m}\right) \eta_{t_{0}}^{\top} R \eta_{t_{0}} \\
& +\tau e^{\alpha \tau} \lambda_{\max }\left(\widetilde{Q}_{m}\right) \sup _{-\tau \leq \theta \leq 0}\left\{\eta_{\theta}^{\top} R \eta_{\theta}\right\} \\
\leq & \left(\lambda_{\max }\left(\widetilde{P}_{i, m}\right)+\tau e^{\alpha \tau} \lambda_{\max }\left(\widetilde{Q}_{m}\right)\right) \sup _{-\tau \leq \theta \leq 0}\left\{\eta_{\theta}^{\top} R \eta_{\theta}\right\} \\
\leq & \left(\lambda_{2}+\tau e^{\alpha \tau} \lambda_{3}\right) \sup _{-\tau \leq \theta \leq 0}\left\{\eta_{\theta}^{\top} R \eta_{\theta}\right\} \\
\leq & \left(\lambda_{2}+\tau e^{\alpha \tau} \lambda_{3}\right) c_{1} .
\end{aligned}
$$

The proof is completed.

Remark 8. It should be noted that the linear feedback control subject to piecewise constant transition probability is first considered in the paper, and it is classical and effective to stabilize the Markov jump system.

\section{Finite-Time $H_{\infty}$ Performance Analysis}

Theorem 9. For a given constant $T>0, \alpha>0$, system (5) is robustly finite-time stochastic boundedness with respect to $\left(0, c_{2}, N, R, d, \sigma\right)$, if there exist positive definite matrices $X_{i, m}$, $Q_{1 m}, Q_{2 m}, H$, and $\mu>1$, such that the following linear matrix inequalities 


$$
\left[\begin{array}{ccccccccc}
\Sigma_{11 i, m} & \Sigma_{12 i, m} & A_{\tau i} Q_{1 m} & 0 & D_{i} & X_{i, m} & 0 & \Sigma_{18 i} & \Sigma_{19 i} \\
* & \Sigma_{22 i, m} & 0 & 0 & 0 & 0 & X_{i, m} & 0 & 0 \\
* & * & -e^{\alpha \tau} Q_{1 m} & 0 & 0 & 0 & 0 & 0 & 0 \\
* & * & * & -e^{\alpha \tau} Q_{2 m} & 0 & 0 & 0 & 0 & 0 \\
* & * & * & * & -\alpha H & 0 & 0 & 0 & 0 \\
* & * & * & * & * & -Q_{1 m} & 0 & 0 & 0 \\
* & * & * & * & * & 0 & -Q_{2 m} & 0 & 0 \\
* & * & * & * & * & * & * & \Sigma_{88 i} & 0 \\
* & * & * & * & * & * & * & * & \Sigma_{99 i}
\end{array}\right]<0,
$$

$$
\begin{gathered}
\forall i, j \in \mathcal{N}, m, n \in \mathscr{M}, \\
X_{i, m} \leq \mu X_{i, n}, \\
Q_{k m} \leq Q_{k n}, \\
(k=1,2) \quad \forall i, j \in \mathcal{N}, m, n \in \mathscr{M}, \\
\alpha c_{2}>d \lambda_{4} e^{\alpha T}\left(1-e^{-\alpha T}\right),
\end{gathered}
$$

where

$$
\begin{aligned}
& \Sigma_{11 i} \\
& =A_{i} X_{i, m}+X_{i, m}^{\top} A_{i}+B_{i} Y_{4 i, m} C_{i}+C_{i}^{\top} Y_{4 i, m}^{\top} B_{i}^{\top}-\alpha X_{i, m} \\
& -\left(N_{i}-1\right) \max \mu_{i} X_{i, m}, \\
& \Sigma_{12 i}=C_{i}^{\top} Y_{2 i, m}^{\top}+B_{i} Y_{3 i, m}, \\
& \Sigma_{22 i}=Y_{1 i, m}+Y_{1 i, m}^{\top}-\alpha X_{i, m} \text {, } \\
& \Sigma_{18 i} \\
& =\Sigma_{19 i} \\
& =[\overbrace{\sqrt{\max \mu_{i}} X_{i, m}, \sqrt{\max \mu_{i}} X_{i, m}, \ldots, \sqrt{\max \mu_{i}} X_{i, m}}^{N_{i}-1}], \\
& \Sigma_{88 i} \\
& =\Sigma_{99 i} \\
& =-\operatorname{diag}\left\{X_{1, m}, \ldots, X_{i-1, m}, X_{i+1, m}, \ldots, X_{N, m}\right\} \text {. }
\end{aligned}
$$

with the average dwell time of the switching signal $\sigma$ satisfying

$$
\tau_{a}>\tau_{a}^{*}=\frac{T \ln \mu}{\ln \left(\alpha c_{2}\right)-\ln \left[d \lambda_{4}\left(1-e^{-\alpha T}\right)\right]-\alpha T},
$$

and the feasible solutions are given as follows:

$$
\begin{aligned}
& A_{f i, m}=Y_{1 i, m} X_{i, m}^{-1}, \\
& B_{f i, m}=Y_{2 i, m} U_{i} S_{i} X_{i, m} S_{i}^{-1} U_{i}^{\top}, \\
& C_{f i, m}=Y_{3 i, m} X_{i, m}^{-1}, \\
& D_{f i, m}=Y_{2 i, m} U_{i} S_{i} X_{i, m} S_{i}^{-1} U_{i}^{\top} .
\end{aligned}
$$

Then the closed-loop systems (5) are finite-time boundedness with respect to $\left(0, c_{2}, T, d, R, \sigma\right)$.

Proof. Pre- and postmultiply inequality (12) by $\operatorname{diag}\left\{P_{i, m}^{-1}\right.$, $I, I\}$, it yields that

$$
\left[\begin{array}{ccc}
P_{i, m} \bar{A}_{i, m}^{\top}+\bar{A}_{i, m} P_{i, m}+P_{i, m} \bar{P}_{i, m} P_{i, m}-\alpha P_{i, m}+P_{i, m} Q_{m}^{-1} P_{i, m} & \bar{A}_{\tau i} & \bar{B}_{i} \\
* & -e^{\alpha \tau} Q_{m}^{-1} & 0 \\
* & * & -\alpha H
\end{array}\right]<0 .
$$


Denote $P_{i, m}=\operatorname{diag}\left\{X_{i, m}, X_{i, m}\right\}, Q_{m}=\operatorname{diag}\left\{Q_{1 m}, Q_{2 m}\right\}$; using Schur complement, we can obtain

$$
\left[\begin{array}{ccccccccc}
\Sigma_{11 i, m} & \Sigma_{12 i, m} & A_{\tau i} Q_{1 m} & 0 & D_{i} & X_{i, m} & 0 & \Sigma_{18 i} & \Sigma_{19 i} \\
* & \Sigma_{22 i, m} & 0 & 0 & 0 & 0 & X_{i, m} & 0 & 0 \\
* & * & -e^{\alpha \tau} Q_{1 m} & 0 & 0 & 0 & 0 & 0 & 0 \\
* & * & * & -e^{\alpha \tau} Q_{2 m} & 0 & 0 & 0 & 0 & 0 \\
* & * & * & * & -\alpha H & 0 & 0 & 0 & 0 \\
* & * & * & * & * & -Q_{1 m} & 0 & 0 & 0 \\
* & * & * & * & * & 0 & -Q_{2 m} & 0 & 0 \\
* & * & * & * & * & * & * & \Sigma_{88 i} & 0 \\
* & * & * & * & * & * & * & * & \Sigma_{99 i}
\end{array}\right]<0,
$$

where

$$
\begin{aligned}
\Sigma_{11 i}= & A_{i} X_{i, m}+X_{i, m}^{\top} A_{i} \\
& +B_{i} D_{f i, m} U_{i}^{-\top} S_{i} X_{1 i, m} S_{i}^{-1} U_{i}^{-1} C_{i} \\
& +C_{i}^{\top} U_{i}^{-\top} S_{i}^{-\top} X_{1 i, m}^{\top} S_{i}^{\top} U_{i}^{-1} B_{i} D_{f i, m}^{\top}-\alpha X_{i, m} \\
& -\left(N_{i}-1\right) \max \mu_{i} X_{i, m}, \\
\Sigma_{12 i}= & C_{i}^{\top} U_{i}^{-\top} S_{i}^{-\top} X_{i, m} S_{i}^{\top} U_{i}^{-1} B_{f i, m}^{\top}+B_{i} C_{f i, m} X_{i, m}, \\
\Sigma_{22 i}= & A_{f i, m} X_{i, m}+X_{i, m} A_{f i, m}^{\top}-\alpha X_{i, m} .
\end{aligned}
$$

Define

$$
\begin{aligned}
& Y_{1 i, m}=A_{f i, m} X_{i, m}, \\
& Y_{2 i, m}=B_{f i, m} U_{i}^{-\top} S_{i} X_{i, m} S_{i}^{-1} U_{i}^{-1}, \\
& Y_{3 i, m}=C_{f i, m} X_{i, m}, \\
& Y_{4 i, m}=D_{f i, m} U_{i}^{-\top} S_{i} X_{i, m} S_{i}^{-1} U_{i}^{-1} .
\end{aligned}
$$

And $X_{i, m} \leq \mu X_{i, n}$ can guarantee that $P_{i, m} \leq \mu P_{i, n}$; then we can obtain (34).

\section{Illustrative Example}

Consider the system as follows:

$$
\begin{aligned}
& B_{1}=\left[\begin{array}{c}
1 \\
0.5 \\
2
\end{array}\right] \text {, } \\
& D_{1}=\left[\begin{array}{l}
0.3 \\
0.5 \\
0.2
\end{array}\right] \text {, } \\
& C_{1}=\left[\begin{array}{lll}
-1.2 & 0.5 & 0.9
\end{array}\right] \text {, } \\
& A_{2}=\left[\begin{array}{ccc}
-1.5 & -1.2 & -1.5 \\
0.2 & -1.5 & 0.4 \\
-0.7 & 1.1 & -1.2
\end{array}\right] \text {, } \\
& A_{\tau 2}=\left[\begin{array}{lll}
0.2 & 0.0 & 0.0 \\
0.1 & 0.2 & 0.1 \\
0.1 & 0.1 & 0.3
\end{array}\right], \\
& B_{2}=\left[\begin{array}{l}
0.5 \\
0.7 \\
1.5
\end{array}\right] \text {, } \\
& D_{2}=\left[\begin{array}{l}
0.4 \\
0.2 \\
0.3
\end{array}\right] \text {, } \\
& C_{2}=\left[\begin{array}{lll}
-1.0 & 1.2 & 0.5
\end{array}\right] \text {. }
\end{aligned}
$$

$$
\begin{aligned}
A_{1} & =\left[\begin{array}{ccc}
-2.0 & -1.5 & -1.2 \\
0.7 & -1.6 & 0.5 \\
-1.3 & 0.5 & -1.1
\end{array}\right], \\
A_{\tau 1} & =\left[\begin{array}{lll}
0.2 & 0.0 & 0.1 \\
0.1 & 0.3 & 0.1 \\
0.3 & 0.1 & 0.2
\end{array}\right],
\end{aligned}
$$

The piecewise-constant transition probabilities matrices are given as

$$
\begin{aligned}
& \Pi^{1}=\left[\begin{array}{cc}
0.1 & -0.1 \\
-0.9 & 0.9
\end{array}\right], \\
& \Pi^{2}=\left[\begin{array}{cc}
0.2 & -0.2 \\
-0.6 & 0.6
\end{array}\right] .
\end{aligned}
$$


Choosing $\alpha=0.05, \tau=0.2, c_{2}=30, T=10$, and $d=0.01$, by solving the matrix equalities in Theorem 9 , we have the following filter parameters:

$$
\begin{aligned}
& A_{f 1,1}=\left[\begin{array}{ccc}
2.6498 & 0.1514 & -3.48944 \\
-1.4622 & -3.1536 & 3.9281 \\
-5.2184 & -3.2024 & -8.9715
\end{array}\right], \\
& B_{f 1,1}=\left[\begin{array}{l}
0 \\
0 \\
0
\end{array}\right] \text {, } \\
& C_{f 1,1}=\left[\begin{array}{lll}
0 & 0 & 0
\end{array}\right], \\
& D_{f 1,1}=-46.3725 \text {, } \\
& A_{f 2,1}=\left[\begin{array}{ccc}
-8.1617 & 10.4852 & 14.5961 \\
-19.9581 & 38.1415 & 64.6288 \\
-10.6012 & 19.4270 & 28.2844
\end{array}\right] \text {, } \\
& B_{f 2,1}=\left[\begin{array}{l}
0 \\
0 \\
0
\end{array}\right], \\
& C_{f 2,1}=\left[\begin{array}{lll}
0 & 0 & 0
\end{array}\right] \text {, } \\
& D_{f 2,1}=-48.6350 \text {, } \\
& A_{f 1,2}=\left[\begin{array}{ccc}
-2.8214 & 1.9349 & 5.6510 \\
6.9619 & -6.9841 & -9.9841 \\
4.3016 & -10.9846 & -17.9894
\end{array}\right] \text {, } \\
& B_{f 1,2}=\left[\begin{array}{l}
0 \\
0 \\
0
\end{array}\right], \\
& C_{f 1,2}=\left[\begin{array}{lll}
0 & 0 & 0
\end{array}\right], \\
& D_{f 1,2}=-48.3364 \text {, } \\
& A_{f 2,2}=\left[\begin{array}{ccc}
-7.2081 & 4.0201 & 11.3047 \\
-6.3204 & 8.6193 & 16.2141 \\
-4.3612 & 17.7841 & 12.9564
\end{array}\right] \text {, } \\
& B_{f 2,2}=\left[\begin{array}{l}
0 \\
0 \\
0
\end{array}\right] \text {, } \\
& C_{f 2,2}=\left[\begin{array}{lll}
0 & 0 & 0
\end{array}\right], \\
& D_{f 2,2}=-46.0053 \text {. }
\end{aligned}
$$

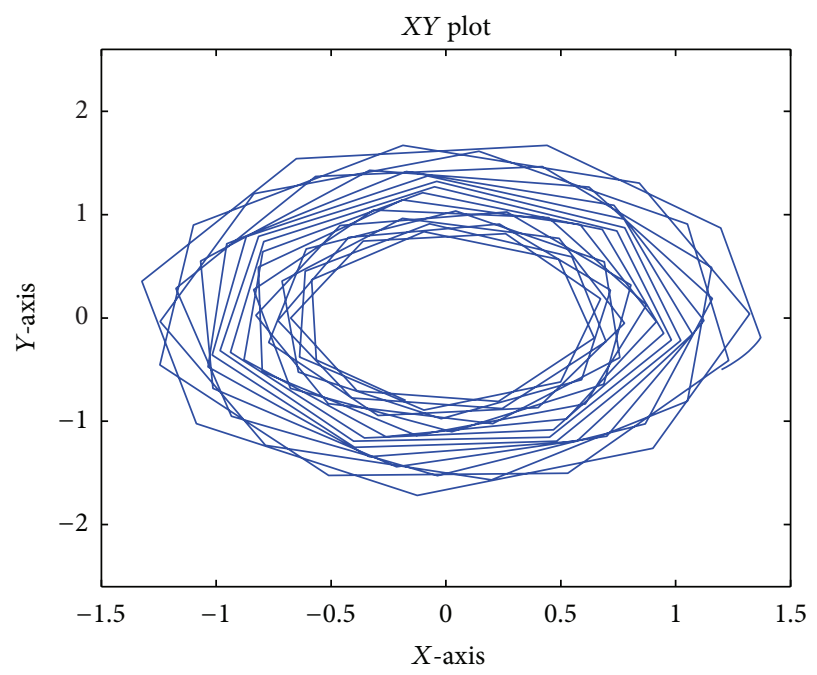

FiguRe 1: $\eta_{t}^{\top} T \eta_{t}$.

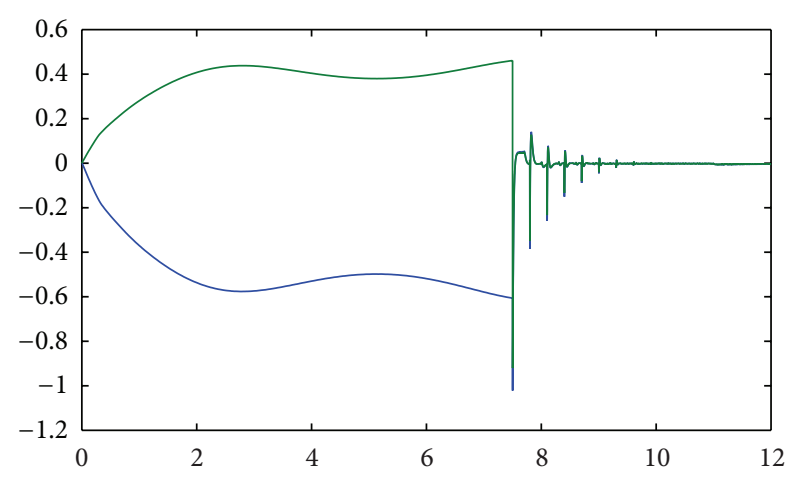

FIGURE 2: State trajectories of subsystem 1.

From (38), we have $\mu=5.0216$. Moreover, we can obtain the average dwell time

$$
\tau_{a}>\tau_{a}^{*}=8.1023 .
$$

By Theorem 9, through the program $f$ minsearch in the optimization toolbox of MATLAB, the optimal bound with minimum value of $c_{2}$ relies on the parameter $\alpha$. We can find feasible solution when $\alpha \in[0,0.05]$. Figure 1 shows the solution trajectory of the system. The state trajectory of the closed-loop system is shown in Figures 2-4, where the initial state $\eta_{0}=[0,0]^{\top}$. From Figures $2-4$, it is easy to see that the system is finite-time boundedness.

\section{Conclusions}

In this paper, the problems of finite-time boundedness of Markovian jump system with piecewise-constant transition probabilities via dynamic output feedback control is concerned. By allowing new Lyapunov-Krasovskii functional, the switching signal is constraint by average dwell time, and a numerical example is also given to demonstrate the effectiveness of the proposed approach. 


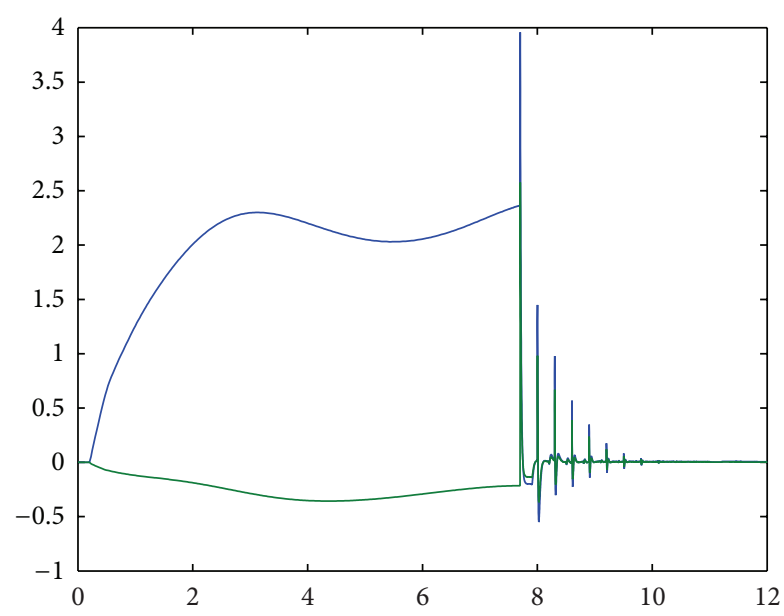

FIGURE 3: State trajectories of subsystem 2.

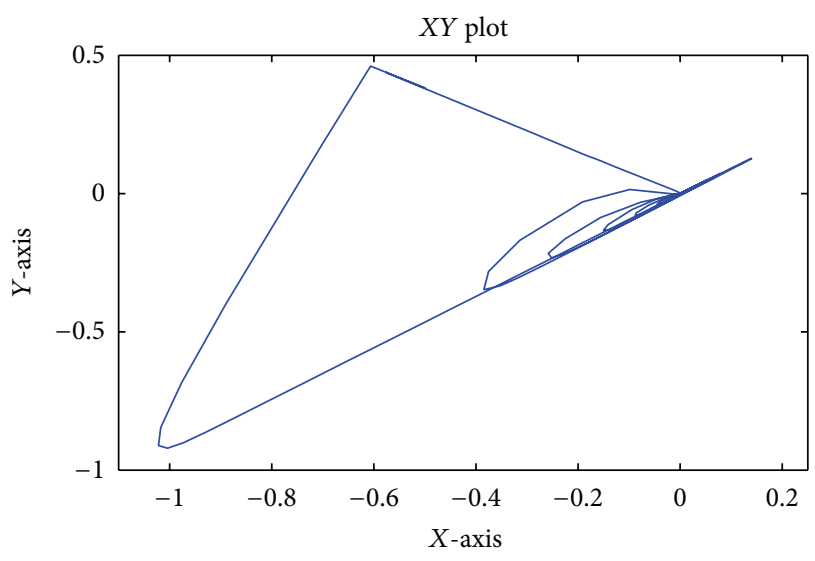

Figure 4: The closed-loop system.

\section{Conflict of Interests}

The authors declare that there is no conflict of interests regarding the publication of this paper.

\section{Acknowledgments}

This work was supported by the National Nature Science Foundation of China (Grant no. 61173121), the Postdoctoral Foundation of China (Grant no. 20100471665), the Youth Science Foundation of Sichuan Province, in China (Grant no. 2010JQ0032), and the Science Foundation of Chengdu City in China (Grant no. 60873092).

\section{References}

[1] N. N. Krasovskii and È. A. Lidskiı̌, "Analytical design of controllers in systems with random attributes. I. Statement of the problem, method of solving," Automation and Remote Control, vol. 22, pp. 1021-1025, 1961.

[2] D. Zhang, W. Cai, L. Xie, and Q. Wang, "Non-fragile distributed filtering for T-S fuzzy systems in sensor networks," IEEE Transactions on Fuzzy Systems, no. 99, 2014.
[3] Q. Zhu, R. Rakkiyappan, and A. Chandrasekar, "Stochastic stability of Markovian jump BAM neural networks with leakage delays and impulse control," Neurocomputing, vol. 136, pp. 136151, 2014.

[4] Q. Zhu, "pth moment exponential stability of impulsive stochastic functional differential equations with Markovian switching," Journal of the Franklin Institute, vol. 351, no. 7, pp. 3965-3986, 2014.

[5] Q. Zhu and J. Cao, "Robust exponential stability of markovian jump impulsive stochastic Cohen-Grossberg neural networks with mixed time delays," IEEE Transactions on Neural Networks, vol. 21, no. 8, pp. 1314-1325, 2010.

[6] Q. Zhu and J. Cao, "Stability analysis of markovian jump stochastic BAM neural networks with impulse control and mixed time delays," IEEE Transactions on Neural Networks and Learning Systems, vol. 23, no. 3, pp. 467-479, 2012.

[7] Q. Zhu, J. Cao, T. Hayat, and F. Alsaadi, "Robust stability of Markovian jump stochastic neural networks with time delays in the leakage terms," Neural Processing Letters, vol. 41, pp. 1-27, 2015.

[8] Q. Zhu and J. Cao, "Exponential stability of stochastic neural networks with both Markovian jump parameters and mixed time delays," IEEE Transactions on Systems, Man, and Cybernetics, Part B: Cybernetics, vol. 41, no. 2, pp. 341-353, 2011.

[9] L. Zhang and E.-K. Boukas, "Stability and stabilization of Markovian jump linear systems with partly unknown transition probabilities," Automatica, vol. 45, no. 2, pp. 463-468, 2009.

[10] L. Zhang and E.-K. Boukas, " $H_{\infty}$ control for discrete-time Markovian jump linear systems with partly unknown transition probabilities," International Journal of Robust and Nonlinear Control, vol. 19, no. 8, pp. 868-883, 2009.

[11] M. Misas and M. T. Ramirez, "Colombian economic growth under Markov switching regimes with endogenous transition probabilities," Borradores de Economía, vol. 425, pp. 1-21, 2006.

[12] T. C. Cheng, C. Gao, and H. Shen, "Production and inventory rationing in a make-to-stock system with a failure-prone machine and lost sales," IEEE Transactions on Automatic Control, vol. 56, no. 5, pp. 1176-1180, 2011.

[13] A. J. Filardo, "Business-cycle phases and their transitional dynamics," Journal of Business and Economic Statistics, vol. 12, no. 3, pp. 299-308, 1994.

[14] L. Zhang, Y. Leng, L. Chen, and Y. Zhao, "A BRL for a class of discrete-time Markov jump linear system with piecewiseconstant TPs," in Proceedings of the 18th IFAC World Congress, pp. 8699-8704, Milano, Italy, September 2011.

[15] F. Takeuchi, "US external debt sustainability revisited: bayesian analysis of extended Markov switching unit root test," Japan and the World Economy, vol. 22, no. 2, pp. 98-106, 2010.

[16] S. Boyd, L. El Ghaoui, E. Feron, and V. Balakrishnan, Linear Matrix Inequalities in System and Control Theory, vol. 15 of SIAM Studies in Applied Mathematics, SIAM, Philadelphia, Pa, USA, 1994.

[17] Z. Wu, H. Su, and J. Chu, "State estimation for discrete Markovian jumping neural networks with time delay," Neurocomputing, vol. 73, no. 10-12, pp. 2247-2254, 2010.

[18] Q. Zhu and J. Cao, "Adaptive synchronization under almost every initial data for stochastic neural networks with timevarying delays and distributed delays," Communications in Nonlinear Science and Numerical Simulation, vol. 16, no. 4, pp. 2139-2159, 2011. 
[19] L. Chen, Y. Leng, H. Guo, P. Shi, and L. Zhang, " $H_{\infty}$ control of a class of discrete-time Markov jump linear systems with piecewise-constant TPs subject to average dwell time switching," Journal of the Franklin Institute, vol. 349, no. 6, pp. 19892003, 2012.

[20] L. Zhang and B. Jiang, "Stability of a class of switched linear systems with uncertainties and average dwell time switching," International Journal of Innovative Computing, Information and Control, vol. 6, no. 2, pp. 667-676, 2010.

[21] L. Zhang, E.-K. Boukas, and P. Shi, "Exponential $H_{\infty}$ filtering for uncertain discrete-time switched linear systems with average dwell time: a $\mu$-dependent approach," International Journal of Robust and Nonlinear Control, vol. 18, no. 11, pp. 1188-1207, 2008.

[22] F. Amato, M. Ariola, and P. Dorato, "Finite-time control of linear systems subject to parametric uncertainties and disturbances," Automatica, vol. 37, no. 9, pp. 1459-1463, 2001.

[23] F. Amato and M. Ariola, "Finite-time control of discrete-time linear systems," IEEE Transactions on Automatic Control, vol. 50, no. 5, pp. 724-729, 2005.

[24] Y. Shen and H. Liu, "Finite-time stabilization of switched timedelay system via dynamic output feedback control," Advances in Intelligent and Soft Computing, vol. 125, pp. 523-528, 2012.

[25] Y. Hong, "Finite-time stabilization and stabilizability of a class of controllable systems," Systems and Control Letters, vol. 46, no. 4, pp. 231-236, 2002.

[26] H. Liu, Y. Shen, and X. Zhao, "Finite-time stabilization and boundedness of switched linear system under state-dependent switching," Journal of the Franklin Institute. Engineering and Applied Mathematics, vol. 350, no. 3, pp. 541-555, 2013.

[27] S. He and F. Liu, "Finite-time $H_{\infty}$ fuzzy control of nonlinear jump systems with time delays via dynamic observer-based state feedback," IEEE Transactions on Fuzzy Systems, vol. 20, no. 4, pp. 605-614, 2012.

[28] J. Cheng, H. Zhu, S. Zhong, Q. Zhong, and Y. Zeng, "Finitetime $H_{\infty}$ estimation for discrete-time Markov jump systems with time-varying transition probabilities subject to average dwell time switching," Communications in Nonlinear Science and Numerical Simulation, vol. 20, no. 2, pp. 571-582, 2015.

[29] X. Luan, F. Liu, and P. Shi, "Finite-time filtering for non-linear stochastic systems with partially known transition jump rates," IET Control Theory and Applications, vol. 4, no. 5, pp. 735-745, 2010.

[30] J. Cheng, L. Xiong, B. Wang, and J. Yang, "Robust finite-time boundedness of $H_{\infty}$ filtering for switched systems with timevarying delay," Optimal Control Applications and Methods, 2015.

[31] X. Luan, F. Liu, and P. Shi, "Robust finite-time $H_{\infty}$ control for nonlinear jump systems via neural networks," Circuits, Systems, and Signal Processing, vol. 29, no. 3, pp. 481-498, 2010.

[32] W. Xiang and J. Xiao, " $H_{\infty}$ finite-time control for switched nonlinear discrete-time systems with norm-bounded disturbance," Journal of the Franklin Institute. Engineering and Applied Mathematics, vol. 348, no. 2, pp. 331-352, 2011. 


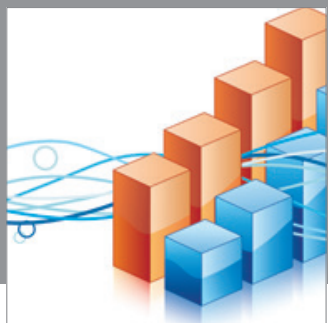

Advances in

Operations Research

mansans

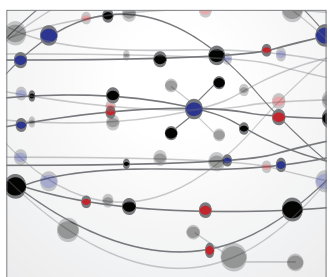

The Scientific World Journal
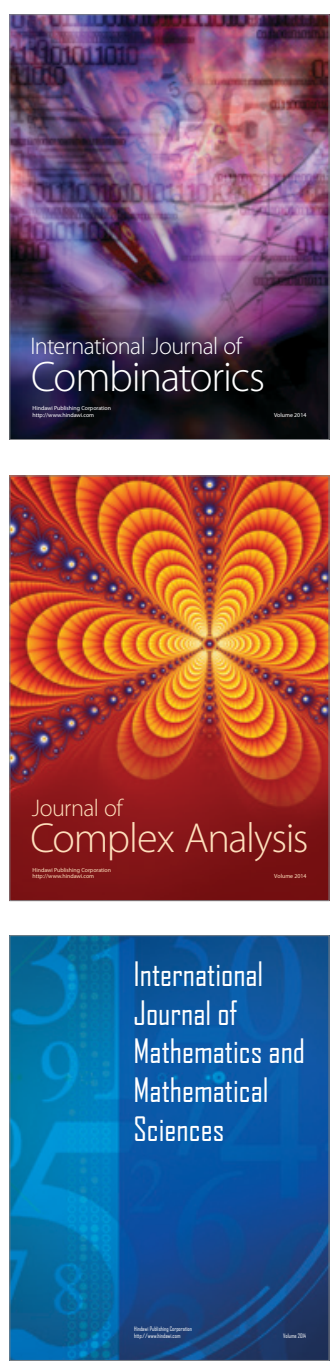
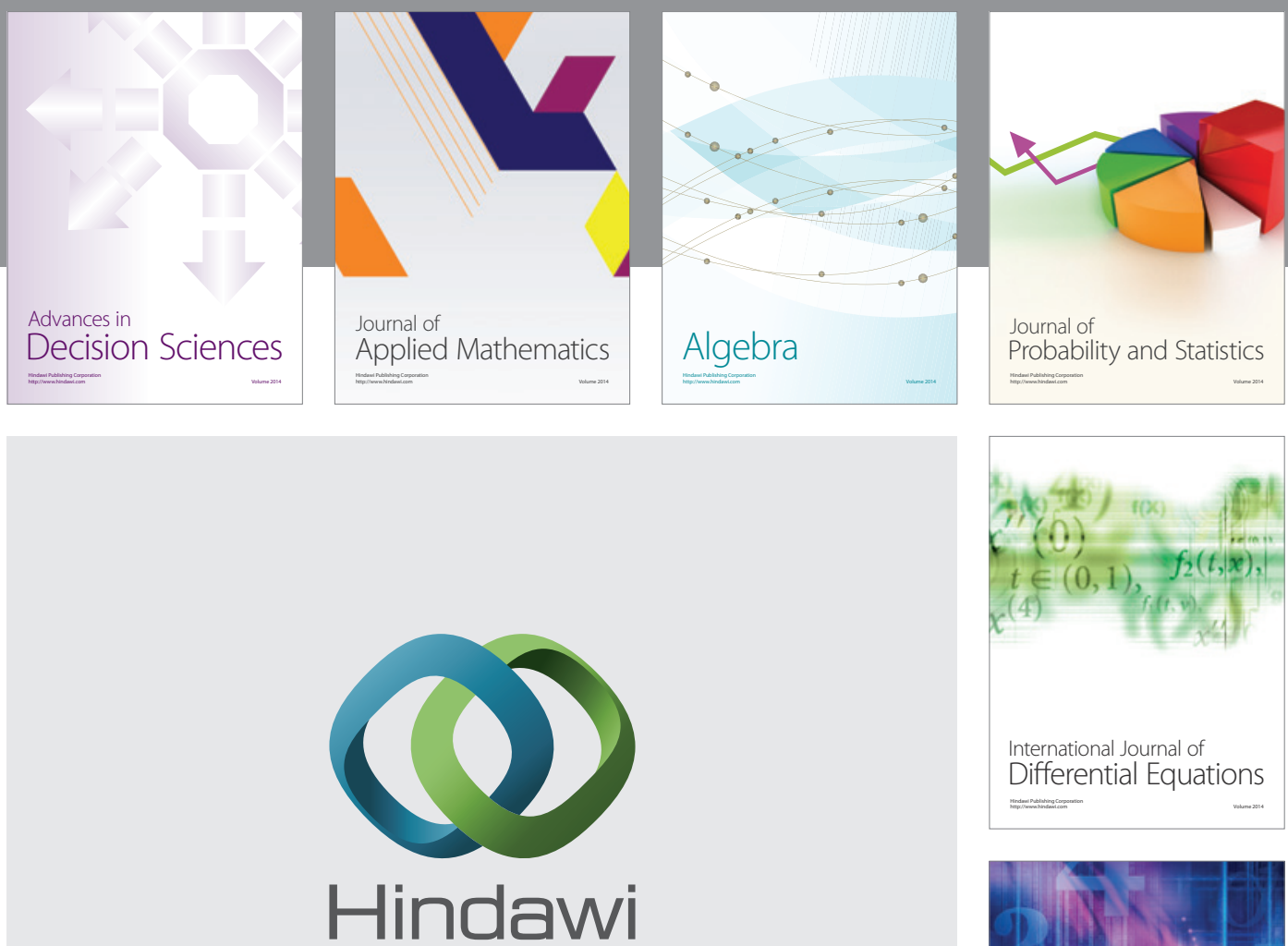

Submit your manuscripts at http://www.hindawi.com
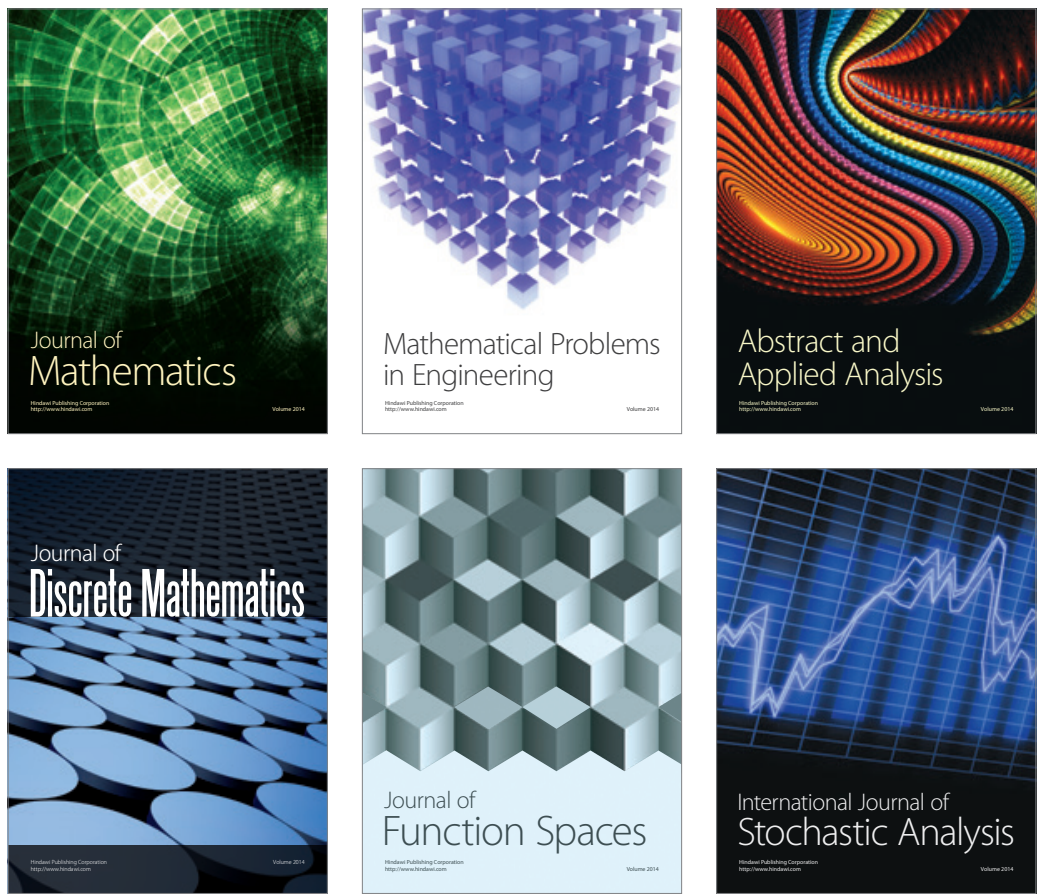

Journal of

Function Spaces

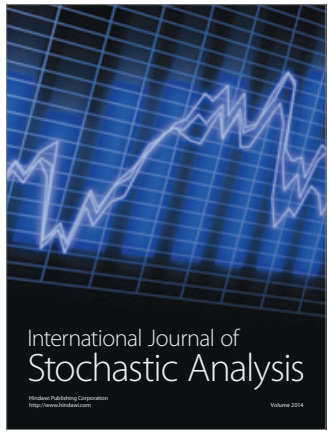

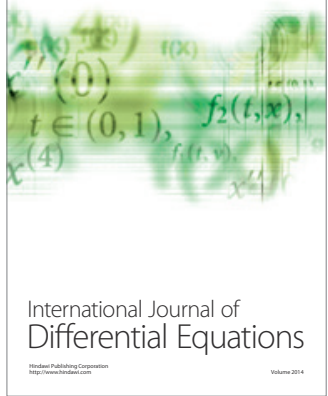
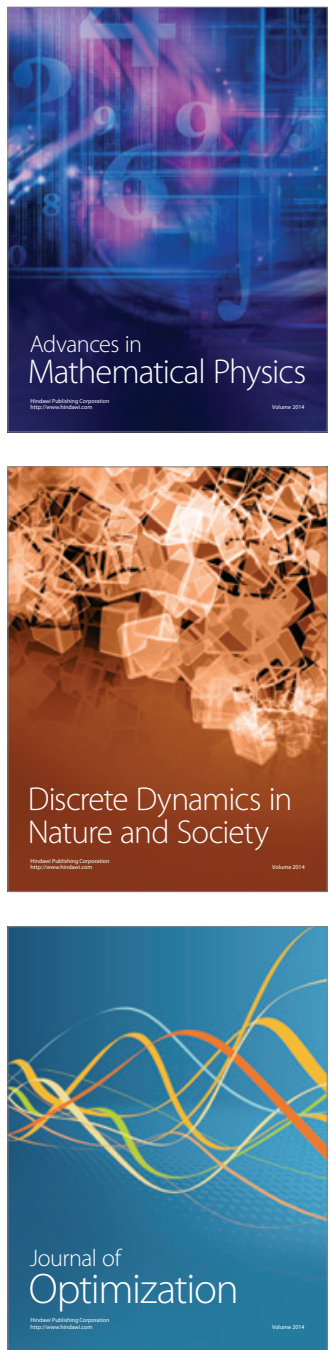